A5

doi: 10.14232/ syrpharmacognosy.2021.a5

\title{
Phytochemical investigation of Carex praecox
}

\section{Zsuzsanna Csilla Dávid}

Email: david.zsuzsanna@pharmacognosy.hu

Cyperaceae species are common members of the flora of the Carpathian Basin. In recent years, species belonging to the genus Carex (Cyperaceae) have attracted attention due to their chemical composition; flavonoids and stilbenoids were identified from some of the species of this genus $[1,2]$.

The aim of our work is the isolation and structure determination of bioactive compounds of Cyperaceae species native to the Carpathian Basin. In the course of this project, Carex praecox Schreb., a small perennial plant native to Europe and western Asia, was investigated.

During the preparative work, the dried and ground whole plant was extracted with methanol and after evaporation it was subjected to solvent-solvent partition with $n$-hexane, chloroform and ethyl acetate. The chloroform fraction seemed to be the most promising based on the preliminary antibacterial tests and TLC investigation. Therefore, it was purified by diverse chromatographic methods, including VLC, MPLC, preparative TLC and HPLC. The structure elucidation of the isolated compounds was carried out by NMR and MS spectroscopic methods. Altogether seven compounds have been isolated from the plant so far, among them two novel flavonoids, two novel and one known lignan derivative, an aldehyde and a chromene type metabolite. All compounds have been isolated for the first time from the plant.

Supervisor: Andrea Vasas

\section{Acknowledgements:}

This work was supported by the Economic Development and Innovation Operative Programme GINOP-2.3.2-15-201600012.

\section{References}

[1] Fiorentino A, et al. Biochemical Systematics and Ecology 2008; 36: 691-698.

[2] Li L, et al. Journal of Agricultural and Food Chemistry 2009; 57: 7282-7287. 